\title{
SUPERCONDUCTING RESONATOR AS BEAM-INDUCED SIGNAL PICKUP
}

\author{
S. I. Sharamentov, B. E. Clifft, P. N. Ostroumov, R. C. Pardo, G. P. Zinkann, ANL, Argonne, IL \\ 60439, USA
}

\section{Abstract}

The first demonstration of a superconducting resonator (SCR), installed for beam acceleration, to detect the arrival time of a beam bunch is described. The detecting resonator is operated at very low field level, comparable to the field induced by the beam pulse traversing the cavity so as to maintain an acceptable response characteristic for this high-Q device. Due to this, the RF field in an SCR is always a superposition of a "pure" (or reference) RF and the beam-induced signal. A new method of circular phase rotation (CPR), allowing extraction of the beam phase information from the composite RF field was developed. Arrival time phase determination with CPR is better than one degree for a beam current of $100 \mathrm{nA}$. The electronics design is described and example data are presented.

\section{INTRODUCTION}

Superconducting linear accelerators have become widely popular for the acceleration of all types of charged particles $[1,2]$ due to their high accelerating gradients and low operating costs. The design of these linacs relies on the use of short independently phased cavities with transverse focusing elements interspersed among the resonators. For optimum beam optics, close spacing of the active elements is needed with minimum drift distances. This requirement is especially true in the low-velocity $(E d 0.1)$ accelerator region for heavy-ions with low charge-to-mass ratio $(\mathrm{q} / \mathrm{m})$, and so the pressure to minimize the space needed for diagnostics is intense. In existing heavy-ion linacs such as ATLAS, no space has been allocated for diagnostics over ten meter distances. At velocities under $0.05 \mathrm{c}$, beam steering and focusing problems make longitudinal diagnostics cumbersome and can induce distortions in the data unrelated to the actual beam conditions in the early resonators. This problem will be even more acute in SC linacs planned for future large facilities such as the Rare Isotope Accelerator (RIA) [3]. In this paper, we demonstrate for the first time the use of a superconducting resonator, installed for beam acceleration, to detect the arrival time of a beam bunch at the detecting resonator. This is accomplished when the resonator is operating, not as an accelerating cavity, but rather running with a very low field, comparable to the field induced by the beam pulse traversing the cavity. In this mode the information obtained from the detecting resonator can be used to accurately determine the beam$\mathrm{RF}$ phase relationship in an upstream resonator and thereby correctly set that resonator for the proper accelerating mode.

\section{SCR FEATURES AND BEAM PHASE MEASUREMENTS}

Very narrow frequency bandwidth and microphonic effect do not allow direct use of the induced RF signal in the SCR for beam phase detection. Similar to the normal SCR application for particle acceleration, beam phase detection requires stabilization of the SCR resonant frequency, which is typically accomplished by means of RF feedback loops. Another way of minimizing microphonics is to decrease the Q value of the SCR. This can be done, for example, by overcoupling an SCR drive port. For the SCR application as a beam phase detector, this method cannot be recommended, however, due to the lower sensitivity. To achieve normal operation of the feedback loops, some initial level of RF field inside the resonator is required. The level of the RF field has to be as low as possible. Otherwise the energy of the incoming beam can be changed due to the interaction between the beam and cavity's RF field. Therefore, unlike "normal" beam phase detectors, the RF field in an SCR is always a superposition of a "pure" (or reference) RF and the beaminduced signal:

$$
A_{6}(t) \exp \left(i I_{6}(t)\right) \quad A_{r f} \exp \left(i I_{r f}(t)\right) \square A_{b} \exp \left(i I_{b}\right),
$$

with amplitude $A_{6}(t) \quad A_{r f} \sqrt{1 \square p^{2} \square 2 p \cos \left(I_{r f} \square I_{b}\right)}$

and phase $I_{6}(t) \quad \arctan \frac{\sin I_{r f} \square p \sin I_{b}}{\cos I_{r f} \square p \cos I_{b}}$, where $A_{r f}, I_{r f}$ are the magnitude and phase of the reference RF field, $A_{b}, I_{b}$ are similar parameters for the beaminduced signal, $p \quad A_{b} / A_{r f}$ is the relative beam-induced signal magnitude. For small $p$, the amplitude of the resultant field can be written as

$$
A_{6}(t) \mid A_{r f}\left(1 \square p \cos \left(I_{r f} \square I_{b}\right)\right) .
$$

As follows from these equations, neither amplitude nor phase of the resonator RF field can give unambiguous information about beam phase, because both amplitude and phase of the resultant RF field are functions of the reference RF field amplitude $A_{r f}$ and phase $I_{r f}$. Nevertheless, there is a simple way to extract beam phase information from the resultant RF field. Suppose, we apply a linear circular phase modulation to the reference 
RF field: $I_{r f}(t) \quad Z_{r} t \quad 2 S F_{r} t$, which can be described as a continuous circular rotation of the RF vector with modulation frequency $F_{r}$. Then, the magnitude of the resultant RF field will be amplitude modulated with the same frequency $F_{r}$, and, relative to the reference signal $\cos Z_{r} t$, will be shifted in phase by an angle exactly equal to the beam phase $I_{b}$.

Thus, by applying a linear circular phase rotation to the reference RF field, and measuring the phase shift between the resonator's resultant signal magnitude and modulation signal, one can measure the beam-induced signal phase.

\section{ATLAS BEAM PHASE DETECTION SETUP}

All beam phase-detection tests were made at the first PII cryostat, which has six superconducting quarter-wave resonators with central frequency $48.5 \mathrm{MHz}$. Their position numbers are R111, R112...R116. Figure 1 shows a simplified RF diagram of an ATLAS superconducting resonator in normal accelerating mode. The resonator operates in a self-excited mode, with locked amplitude and phase feedback loops. Phase locking is accomplished by means of a voltage-controlled reactance VCX [4].

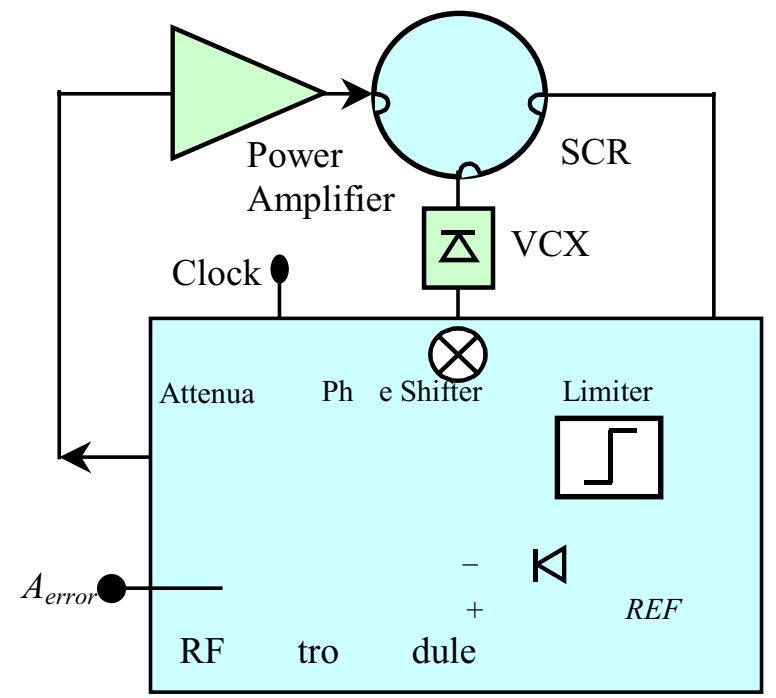

Figure 1. Simplified RF diagram of ATLAS super conducting resonator in normal accelerating mode

The complete experimental setup for using a SCR as a beam phase detector is shown in Figure 2. For low field operation, an additional $40 \mathrm{~dB}$ gain preamplifier and a 20 $\mathrm{dB}$ attenuator were installed in resonator pick-up line and power amplifier input line. The preamplifier and attenuator allow normal operation of the RF control module and RF power amplifier, while the resonator field is only $1 \%$ of the normal accelerating mode. This background field can cause acceleration of the beam in the detecting resonator and affect the effective arrival time in the resonator slightly. For the worst case situation, where the velocity is still very low (E 0.015c) and the prior total voltage is low, the observed phase can be perturbed by as much as $2 q$ For all other cases, this perturbation is less than $0.5 q$

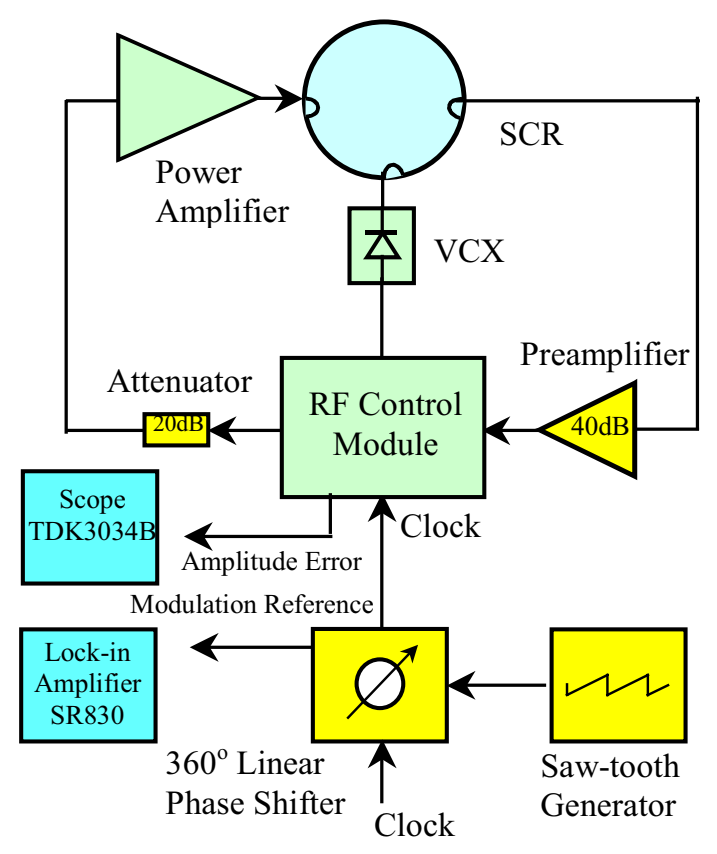

Figure 2. Complete experimental setup for using SCR as beam phase detector.

An external linear phase shifter with $360^{\circ}$ range in the master oscillator (clock) line, controlled by a saw-tooth shape signal, provides continuous circular rotation of the reference RF field vector in the resonator, with a $6-7 \mathrm{~Hz}$ rate. Because of the interaction between the rotating reference RF field vector and the beam-induced RF vector inside the resonator, an amplitude error signal in the amplitude feedback loop was also modulated. It was found that the amplitude error signal is very convenient to use as the beam phase signal, because it reflects any changes in the resonator's RF field magnitude, and can be amplified to any desired level.

A synchronous detection method was chosen for extracting the beam phase information, because it provides high accuracy phase measurements in a noisy environment, which is typically the case for SCR, due to the high level of microphonics. A Stanford Research Lab SR-830 two-channel lock-in amplifier was used as the synchronous detector, and a Tektronix digital TDK3034B scope was chosen for waveform display. Overall accuracy of the beam phase measurements in the real ATLAS accelerator noise environment is estimated to be better than half a degree.

\section{EXPERIMENTAL RESULTS}

The most difficult region of the ATLAS linac to tune with our present surface barrier detector diagnostics is the lowest energy section of the Positive Ion Injector (PII) linac. This is due to steering effects from misalignments as well as asymmetric fields inherent in the resonators and 
the relatively long distance $(\sim 10 \mathrm{~m})$ to the diagnostics region at the end of PII. Therefore, we chose to focus on this very low-velocity region of the linac to develop and test this new system.

First beam phase measurements were made using a ${ }^{20} \mathrm{Ne}^{8+}$ beam in March 2002. Electrical beam current was about 1PA. Beam was delivered from the ATLAS ECR injector with a velocity of $0.0085 \mathrm{c}$, and bunched to approximately 0.25 ns FWHM by a two-stage bunching system [5]. The idea was to operate a nearby downstream SCR as the beam phase monitor, as described before, and observe the induced signal phase as a function of the RF phase of an upstream resonator. Figure 3 shows experimental data for the cases where resonators R112, R113 and R114 were alternately used as the beam phase monitor, and the first resonator, R111, was turned on with $\mathrm{RF}$ field at approximately $3 \mathrm{MV} / \mathrm{m}$. The fact that for all three locations of the beam phase monitor, the measured phase curves have a similar shape, and their minimum corresponds to the same value of R111 phase of $300^{\circ}$, is a confirmation that the measured data were real beam phase data. Another important conclusion from the beam phase data in Figure 3 is that the value of R111 RF phase $300^{\circ}$ corresponds to the point of maximum energy gain where the beam and resonator RF relative phase is 0 degrees.

Phase of the Beam Induced Signal as Function of R111 Phase

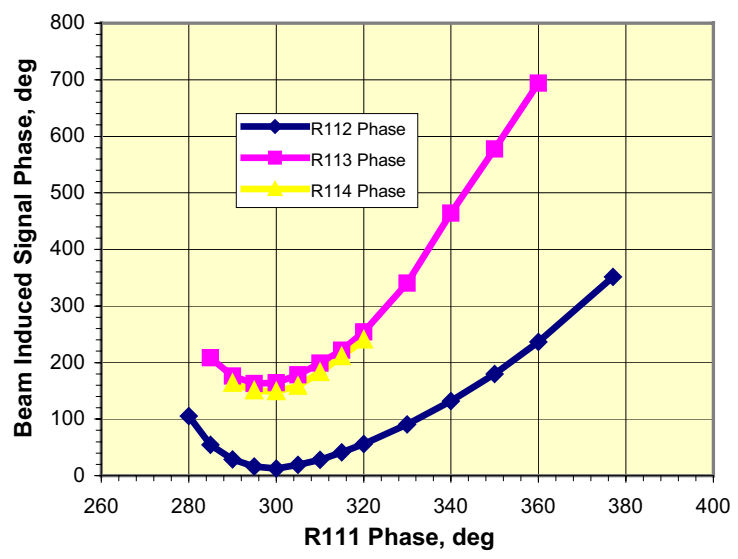

Figure 3. Beam induced signal phase as function of R111 RF phase. Beam phase monitor was placed at three different locations, R112, R113 and R114.

A semi-manual version of this system was then used to 'tune' the first five resonators in the PII linac by sequentially moving the electronics from one resonator to another in order to approximately maintain the velocity match condition and maintain a well bunched beam into the detecting resonator. A wide range of tunes was developed to match beams with charge-to-mass ratio (q/A) from 0.15 to 0.375 . In each instance the actual energy gain was determined using a solid-state detector to measure the absolute energy gain from each resonator. In principle, the total phase shift observed at the detecting resonator between the last accelerating resonator in 'on' and 'off' states is the beam transit time change due to the energy gain given to the beam by that resonator. The energy gain of the beam is calculable within that model and the total beam energy at that point can be determined. The measured total phase shift and the calculated energy of the beam after acceleration are presented in Table I for an ${ }^{40} \mathrm{Ar}^{6+}$ beam. Table I shows data identified by the last accelerating resonator and the detecting resonator. The energy of the ${ }^{40} \mathrm{Ar}$ beam into the first of this set of resonators is $5.5 \mathrm{MeV}$.

If two resonators are used in detection mode, then the difference in phase contains time-of-flight information, which can be used to obtain the absolute energy directly. A calibration procedure would first need development in order to determine the electronic phase offset between the two detecting resonators.

Table 1. Comparison of Phase-Shift Energy Determination and Solid State Detector Measured Energy.

\begin{tabular}{|c|c|c|c|c|}
\hline $\begin{array}{c}\text { Resonator } \\
\text { Pair } \\
\text { (Accel./Det.) }\end{array}$ & $\begin{array}{c}\text { Distance } \\
(\mathrm{m})\end{array}$ & $\begin{array}{c}\text { Total } \\
\text { Phase } \\
\text { Shift } \\
(\mathrm{deg}) /(\mathrm{ns})\end{array}$ & $\begin{array}{c}\text { Energy } \\
\text { Out } \\
(\mathrm{MeV})\end{array}$ & $\begin{array}{c}\text { SBD } \\
\text { measured } \\
\text { energy } \\
(\mathrm{MeV})\end{array}$ \\
\hline R113/ R114 & .540 & $221 / 12.7$ & 7.2 & 7.7 \\
\hline R114/ R116 & .991 & $616 / 35.3$ & 12.6 & 12.7 \\
\hline R115/ R116 & .406 & $104 / 6.0$ & 16.2 & 16.5 \\
\hline
\end{tabular}

\section{CONCLUSION}

The system described in this paper is now in regular use at the ATLAS facility for tuning the resonators in the lowest energy section of the facility. The system is a significant improvement over the previous diagnostics system located 8 meters downstream from these early resonators. Full integration of the method into the ATLAS control system is now underway. Improved phase sensitivity at higher energy may be possible where the technique can be used at the zero-crossing, $90^{\circ}$, point where the maximum possible phase sensitivity exists. At the low velocities studied in this report, the debunching in that phase region makes the detecting resonator insensitive to the beam bunches.

This work was supported by the U.S. Department of Energy under contract W-31-109-ENG-38.

\section{REFERENCES}

[1] L. M. Bollinger, et al., Nucl. Instrum. and Methods, B79, 753(1993).

[2] R. L. Custom, "An Overview of the Spallation Neutron Source Project", Proc. of the XX International Linac Conf., August 21-25, 2000, Monterey, CA, p. 321.

[3] P. N. Ostroumov, J. A. Nolen, and K. W. Shepard, Proceedings of the XX International Linac Conference, August 21-25, 2000, Monterey, CA, p. 1018.

[4] J. M. Bogaty, B. E. Clifft, K. W. Sheppard and G. P. Zinkann, Proceedings of the IEEE Particle Accelerator Conference, 1989, p.1978.

[5] P. K. Den Hartog, J. M. Bogaty, L. M. Bollinger, B. E. Clifft, R. C. Pardo and K. W. Sheppard, Proceedings of the IEEE Particle Accelerator Conference, 1989, p. 545. 